\title{
LA PASSIONE DI CRISTO
}

\author{
Luigi Castagnola \\ Universidade do Paraná
}

Tunc congregati sunt principes sacerdotum, et seniores popull, in atrium principls sacerdotum, qui dicebatur Caiphas: et consilium fecerunt ut Jesum dolo tenerent et occlderent.

S. Matthaeus, XXVI, 3-4.

\section{I}

Sta nel bosco d'ulivi il buon Profeta

E riga il sasso di sudor di sangue.

Non è più questa delle palme l'ora,

Nè delle grida oneste dei fanciulli.

Ora si tratta di salvare il mondo.

Miseramente, nelle scure vie

Ancor sonanti degli evviva a Cristo,

Immondo giace il lutulento ramo,

Sotto le zampe dei cavalli infranto.

Candida brilla, sù nel ciel, la luna.

\section{II}

Sbuca, improvvisa, da Gerusalemme

La turba oscena. La notturna lampa

Di freddo sangue si colora il volto.

Uno li guida, sotto l'ombre rotte,

Come temente di sinistri scheltri

La man gelata. Hanno catene al braccio,

Hanno le torce di vapor fumanti.

Irrequieti sostano. Una voce

Laconica parlò: - Dov'è il Maestro?

Io sono - ei disse. E si lasciò legare. 


\section{III}

Ahi! Gesù profeta abbandonato

Del sommo Caifa nelle man crudeli,

Come una pura vittima propizia.

Nella gran sala lucido si specchia

Sul freddo marmo il colonnato eretto,

Immoto segno d'equità giudea.

Quivi, in giudizio tumultuoso, Caifa,

II pontefice sommo, il sinedrita,

Con tutto dei periti inclito il gruppo,

Siede, mitrato giudice, a lo scanno.

\section{IV}

Siede e dice: - Tu dinne il vero e presto.

Sei tu del Dio il profetato Figlio

Al comando segui breve silenzio.

- S'io ve lo dico non mi crederete -

Rispose il buon Profeta. - Io ti scongiuro -

Caifa principe disse - per Dio vivo:

Sei tu il Cristo promesso a noi venturo?

Brevi parole parla e veritiere

Del pontefice sommo nel cospetto.

\section{$\boldsymbol{\nabla}$}

- Io lo sono - Gesù profeta aggiunse,

$\mathrm{E} \mathrm{mi}$ vedrete giudice fra nubi.

- O sinedriti - e si stracciò le vesti -

Empia bestemmia, o cittadini, udiste.

A voi la scelta libera commetto.

Voi giudicate se impunito io lasci

Questo di leggi sovversore insigne,

$O$ se di morte lo castighi acerba,

Io delle leggi vindice supremo.

- Di morte acerba, disser tutti, e in croce. 
VI

Deh! come pianse sul delitto il sole

Quand' esso nacque a illuminar dall'alto

Di tutti quanti il più ferale giorno,

Piovendo i mesti raggi mattinali

Sui levigati marmi del Pretorio.

Qui fu condotto con le spinte oscene

Il buon Profeta della Galilea

Fra una turba scomposta e sobillata,

Chè alla pena suprema inviar per legge

De l'alma Roma dritto unico è solo.

\section{VII}

Nella purpurea clamide ravvolto

Il console romano a tutti il mostra

In moribondo stato: - Ecco l'uomo.

Io non rinvengo in lui colpa nessuna,

Disse. $\mathbf{E}$ il fremente popolo gridò:

- Di morte è reo, a lui il morir s'addice.

- Sia. Ma innocente io sono. Su di voi

Scenda il suo sangue e sopra i vostri figli.

$\mathbf{E}$, fattosi portare un gran bacile,

Nell'acqua pura l'empie mani asterse.

\section{VIII}

Ah giustizia di Roma e di Pilato!

D'urla selvagge risonâr le strade.

Come se d'oste che a battaglia corre.

I guerrieri, in forbite armi recinti

Ed in pennacchi splendidi, il Profeta

Seguivano con passo abbandonato.

$\mathrm{Ne}$ la tortuosa via l'incontrava

Anche la Madre Vergine, Maria.

Fra le piangenti donne ella rimase

Come di marmo bianca statua appare. 
$\mathbf{D}$

E giunsero sul colle redentore.

I sonanti martelli, in breve tempo,

$\mathrm{Da}$ ben gagliarda forza maneggiati,

II buon Profeta affissero alla croce.

Alzarono quell'opra, unica al mondo,

I nostri mali perfidi, o mortali.

Ed un mistero è questo. Si gridava:

- Salva, salva, o Profeta, ora te stesso.

Mentre, ai suoi piedi avvolte, il Crocifisso

Di puro sangue le pie donne asperse.

\section{$\mathbf{x}$}

Con forte voce Gesù Cristo disse:

- Tutto è compiuto! - E reclinava il capo.

Di terremoti e fiamme arsero i colli

$E$ il sole rosso cuprëo brillò.

In due, nel tempio, il velo si divise.

I cadaveri scossi entro gli avelli,

Come per tuono bamboli dal sonno,

Del centurione confermaro il detto:

- Un uomo giusto veramente egli era.

\section{XI}

Poi che fu a tutti certa la sua morte,

Cuori pietosi lo levâr di croce

E con pietà lo posero nel grembo

De la Vergine Madre dolorosa.

Ahi, passion di Cristo e di Maria!

Diede a quel corpo, con materno affetto,

Un bacio santo. Con pietosa mano,

Svelse le spine in capo, ad una ad una,

Facendo adagio, per non fargli male.

Poi riposò tre dì nel cavo speco. 


\section{XII}

Deh! come fulse d'alma Iuce il sole

Il dì che vide smossa la gran pietra

Che al monumento sacro era custodia.

Morì Gesù profeta e poi risorse

Di luce limpidissima adornato.

Il biondo Galileo, le trecce fulve,

Sul Campidoglio in bianca veste escese,

Di Roma eterna sui ridenti colli.

Delle marmoree deità in eterno

Vittrice, o Roma delle genti, salve!

Curitiba, Sexta-Feira Santa de 1954 